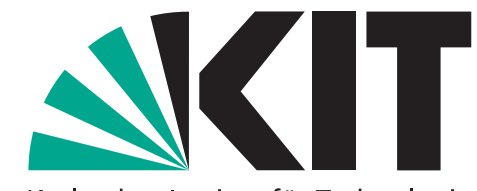

Karlsruher Institut für Technologie

\title{
Revenues and welfare in auctions with information release
}

by Nikolaus Schweizer and Nora Szech

No. 67 | APRIL 2015

\section{WORKING PAPER SERIES IN ECONOMICS}

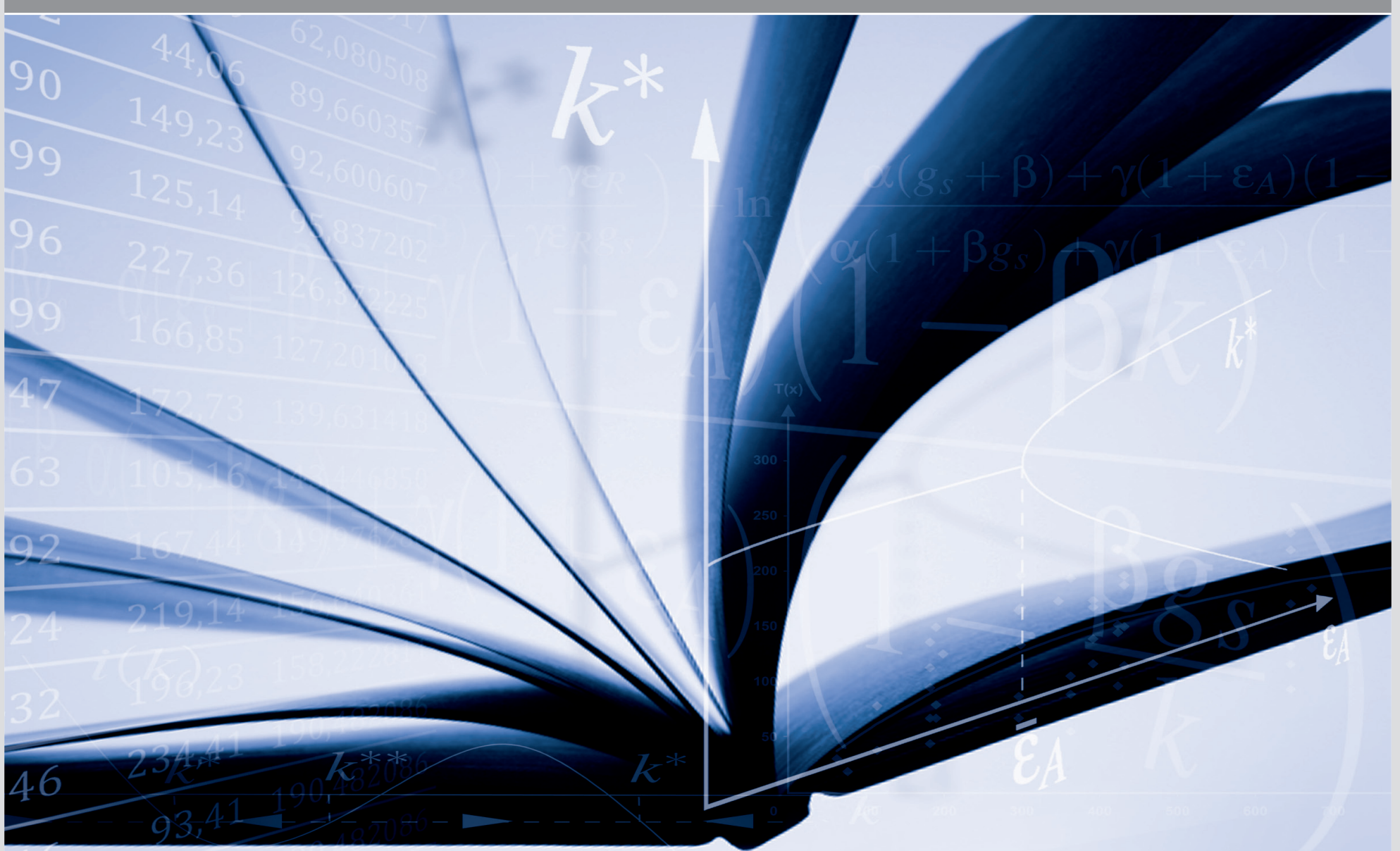




\section{Impressum}

Karlsruher Institut für Technologie (KIT)

Fakultät für Wirtschaftswissenschaften

Institut für Volkswirtschaftslehre (ECON)

Schlossbezirk 12

76131 Karlsruhe

KIT - Universität des Landes Baden-Württemberg und nationales Forschungszentrum in der Helmholtz-Gemeinschaft

Working Paper Series in Economics

No. 67, April 2015

ISSN 2190-9806

econpapers.wiwi.kit.edu 


\title{
Revenues and Welfare in Auctions with Information Release*
}

\author{
Nikolaus Schweizer and Nora Szech
}

April 2015

\begin{abstract}
Auctions are the allocation-mechanisms of choice whenever goods and information in markets are scarce. Therefore, understanding how information affects welfare and revenues in these markets is of fundamental interest. We introduce new statistical concepts, $k$ - and $k$ - $m$-dispersion, for understanding the impact of information release. With these tools, we study the comparative statics of welfare versus revenues for auctions with one or more objects and varying numbers of bidders. Depending on which parts of a distribution of valuations are most affected by information release, welfare may react more strongly than revenues, or vice versa.
\end{abstract}

JEL Classification: D44, D82

Keywords: Auctions, Information Release, Information Partitions, Order Statistics, Stochastic Orders, Dispersion, Dispersive Order, Excess Wealth Order

${ }^{*}$ We would like to thank Benny Moldovanu. Nikolaus Schweizer, Mercator School of Management, Duisburg-Essen University, email: nikolaus.schweizer@uni-due.de. Nora Szech, Chair of Political Economics, Department of Economics, Karlsruhe Institute of Technology, Berlin Social Science Center, and CESifo, email: nora.szech@kit.edu. 


\section{Introduction}

Auctions improve allocations in markets in which information is scarce. They are not only applied in highly capitalized spectrum and timber markets, but also in selling various items from used cars to fine arts. Auctions also serve as models of competition for prizes such as college admissions, winning districts in elections, or finishing among the first in $R \& D$ races. As they are known to allocate scarce goods well despite a fundamental lack of information about the bidders' costs or valuations, it is surprising how little we know about the interaction of information, welfare and revenues in these contexts. This is the starting point of our paper.

Generating information is typically a costly endeavor. For a welfare maximizer, the incentives to provide information on the goods for sale may be very different from the incentives a revenue-maximizing seller faces. The reason behind is that a welfare maximizer incorporates bidders' aggregated rents into his calculation, while a revenue-maximizing seller focuses on the selling price. A priori, releasing information could increase competition at the top such that bidders' rents become smaller. This may affect selling prices a lot, but increase overall efficiency of allocation and thus welfare only marginally. Yet information release could also lead to a further differentiation of the bidders with the highest valuations, thus affecting and increasing bidders' rents and welfare more strongly than the seller's revenue.

Understanding how welfare and revenue incentives relate to each other requires a thorough understanding of the behavior of order statistics. In case of a one-object auction, the first and second order statistics, i.e. the highest and the second highest valuations, and the difference between the two, are crucial. In multi-object auctions, more of the highest order statistics are relevant. If several prizes, like grants or promotions, are "auctioned off" to applicants in order to reward those who exert the highest efforts (bids), efforts of several applicants scratching the top matter. For example, Harvard University selected 2,000 students out of 34,000 applicants for its class of $2018 .^{1}$

The related literature so far focuses on one-object auctions and has mostly imposed conditions on the effects of information release which guarantee that welfare reacts more sensitively towards information than revenue (compare Ganuza and Penalva,

\footnotetext{
${ }^{1}$ See https://college.harvard.edu/admissions/admissions-statistics.
} 
2010). Yet the opposite conclusion can hold true as well. Bidders' rents may decrease in response to information release due to fiercer competition at the top. This implies that a revenue maximizer has stronger incentives to release information than a welfare maximizer. For instance, this is the case when information release affects bidders with intermediate valuations more strongly than bidders with high valuations.

This paper provides criteria that determine whether information release strengthens or weakens competition in multi-object auctions. The previous literature has typically modeled information release as an increase in the variability of valuations in the sense of the dispersive order (Ganuza and Penalva, 2010). Under the dispersive order, additional information always weakens competition as it increases the differences between all order statistics.

We introduce two new classes of stochastic orders that allow for a more flexible and directed control of the behavior of order statistics, the $k$-and $k$-m-dispersion orders. Increased variability in the sense of $k$-dispersion implies that the $k$ highest order statistics move further apart through information release. Increased variability in the sense of $k$ - $m$-dispersion implies the same conclusion when the overall number of bidders $n$ is sufficiently large, $n>k+m$. Both orders are weaker than the dispersive order. In particular, information release can either increase or decrease the variability of valuations in $k$-m-dispersion, implying either a strengthening or a softening of competition. Consequently, a welfare maximizer may have either stronger or weaker incentives to release information than a revenue maximizing seller. $k$ - $m$-dispersion provides a criterion to decide which of the two is the case.

We apply our theory to auctions in which information release is modeled in terms of information partitions. Bidders do not know their true valuations, yet they know which interval of a distribution contains their valuation. Information release renders these intervals finer. This is a prominent model of information release in economic theory (see Bergemann and Pesendorfer, 2007) that is not tractable with the dispersive order. $k$-m-dispersion enables us to draw clear conclusions about multi-object auctions with sufficiently many bidders. Information release decreases bidders' rents if and only if information affects the bidders with the highest valuations.

In a second classical model of information release due to Lewis and Sappington (1994), each bidder's signal equals his valuation with some probability while it is 
pure noise otherwise. This model has been applied to auctions, e.g., by Ganuza and Penalva (2010) and Shi (2012). $k$-m-dispersion allows to determine the comparative statics of information release even if signal quality differs for bidders with higher versus lower valuations. While information release often relaxes competition between bidders in this setting, the opposite can happen as well. Specifically, further increases in high signal qualities may foster competition at the top.

Our techniques also apply to other contexts such as reliability theory and risk management where worst realizations of distributions matter. Differences between order statistics are also crucial in matching markets. Analyzing expected matches between firms and workers, or men and women, requires to control distances between order statistics not only at the top, but also on lower levels of a distribution. Another field of application - beyond the scope of this paper - may be the measurement of inequality, where distances from the poorest (or the richest) to the middle income quantiles of a population may be of specific interest. For example, recent developments in Western countries such as the US suggest that a focus on the distances between the richest 400 families and the middle class could help defining educational goals for the next decades. ${ }^{2}$

\section{Related Literature}

This paper is related to several contributions in the literatures on auctions and on stochastic orders. ${ }^{3}$ Our auction-theoretic applications generalize results of Ganuza and Penalva (2010) and thus contribute to the literature on information in auctions and mechanism design. ${ }^{4}$ Jia, Harstad and Rothkopf (2010) study information release in auctions when bidders know parts of their valuations and the other additive parts can be disclosed. They illustrate that the comparative statistics of bidders' revenues are intricate and conclude that "no illuminating necessary condition seems possible". This is the problem we address. Stochastic orders, especially the dispersive order, have also been applied to study other questions concerning auctions and related contexts, see, for instance, Johnson and Myatt (2006), Mares and Swinkels (2014), Kirkegaard (2014), and the references therein.

In the literature on stochastic orders, parts of our analysis build on a result of $\mathrm{Li}$ and Shaked (2004) who prove one of the main properties of the $k$-dispersion order

\footnotetext{
${ }^{2}$ See "America's elite. An hereditary meritocracy", The Economist, 01/24/2015.

${ }^{3}$ For introductions to these two fields, see Krishna (2002) and Shaked and Shanthikumar (2007).

${ }^{4}$ For a survey, see Bergemann and Välimäki (2006).
} 
without explicitly introducing this order. ${ }^{5}$ We provide new insights on $k$-dispersion and introduce the generalized $k$-m-dispersion orders. As the $k$-dispersion order coincides with the excess wealth order in the case $k=1$, our results are also related to two contributions from the operations research literature which apply the excess wealth order to auctions, $\mathrm{Li}$ (2005) and $\mathrm{Xu}$ and $\mathrm{Li}$ (2008). Analyzing the case $k>1$ allows us to address many questions which are not tractable under the excess wealth order. Paul and Gutierrez (2004) provide several results related to ours based on the star order. Yet their results stating that differences of order statistics can be controlled in terms of the star order are incorrect as is shown in $\mathrm{Xu}$ and $\mathrm{Li}$ (2008). ${ }^{6}$ The same remark applies to Rösler (2014) whose work partially builds on the same incorrect result. Our results can help to alleviate these issues.

\section{Outline}

Section 2 introduces our model and discusses the scope and limitations of modeling information release in terms of the dispersive order. Section 3 introduces our new stochastic orders as well as their key properties. Section 4 presents our main results on information release in multi-object auctions, first in the general case and then in the applications of information partitions and heterogeneous signal quality. Section 5 sketches further economic applications of our methods and presents additional properties of $k$-dispersion. All proofs are in the appendix.

\section{The Setting}

\subsection{Auction Model with Information Release}

We study a symmetric independent private values auction model with information release. Our techniques will allow us to handle one object as well as $k$ object auctions. We therefore introduce the broader setting straight away.

A risk-neutral seller auctions off $k$ identical objects in a $(k+1)^{t h}$ price auction. The $n>k$ bidders are all risk-neutral. Those who submit the $k$ highest bids receive an object and each of them pays the $(k+1)^{t h}$ highest bid. Ties are broken with uniform randomness.

\footnotetext{
${ }^{5}$ Compare Proposition 2.

${ }^{6}$ This incorrect result is cited in Shaked and Shanthikumar (2007) as Theorem 4.B.19 and as Lemma 1 and Lemma 4 in Rösler (2014).
} 
Initially, bidders do not know their valuations exactly. Before the auction takes place, the seller decides whether he wants to release information to the bidders. If he opts against information release, the bidders stick to their initial private estimates $Y_{i}$ of their valuations. The $Y_{i}$ are nonnegative and independently distributed according to a commonly known cumulative distribution function $G$ with finite mean. If the seller opts for information release, each bidder receives an independent signal that reveals more about his valuation for winning an object. We denote by $X_{i}$ the updated estimates of valuations. The random variables $X_{i}$ are again nonnegative, independent and identically distributed with finite mean and we denote their cumulative distribution function by $F . F^{-1}$ and $G^{-1}$ denote the generalized inverse (quantile) functions of $F$ and $G$.

Throughout we assume that all bidders follow their weakly dominant strategy of bidding their best estimate of their valuation in the auction. Thus, bidder $i$ bids $X_{i}$ if information is released and $Y_{i}$ otherwise. We denote by $X_{i: n}$ the $i^{\text {th }}$ order statistic, i.e., the $i^{\text {th }}$-largest out of $X_{1}, \ldots, X_{n}$, and define $Y_{i: n}$ analogously. Lemma 1 summarizes the main properties of the bidding equilibrium.

Lemma 1 Set $Z=X$ if information is released and $Z=Y$ if no information is released. The expected selling price in the auction is given by $E\left[Z_{k+1: n}\right]$. The seller's expected payoff is given by $k E\left[Z_{k+1: n}\right]$. Bidders' aggregate rents are given by

$$
\sum_{j=1}^{k} E\left[Z_{j: n}-Z_{k+1: n}\right]
$$

and total welfare amounts to

$$
\sum_{j=1}^{k} E\left[Z_{j: n}\right] .
$$

In the following, we call the seller a welfare maximizer if he is interested in maximizing total welfare, and we call him a revenue maximizer if he maximizes his expected payoff.

An alternative interpretation of the model is that $F$ denotes a finer information structure compared to $G$, and the seller decides whether to release a signal implementing $G$ or $F$. In the context of information release with Bayesian updating, it is plausible to assume that $F$ and $G$ share the same mean. Our analysis, however, does not rely on this assumption, thus incorporating the possibility of non-Bayesian 
updating by the bidders. As a final interpretation, the seller could decide between running the auction with bidders from two different populations with respective distributions $F$ versus $G$.

We do not impose that $F$ and $G$ are continuous. This allows us to provide results for models of information release such as information partitions that would violate a continuity requirement. The additional structures introduced in Ganuza and Penalva (2010) in the one object case - a prior distribution of valuations, a continuous family of signals with associated costs of information provision, and a continuous family of (posterior) distributions of valuations - directly translate to our setting. In particular, while we do not explicitly specify costs of information release, the comparison between $F$ and $G$ should be thought of as one side of a cost-benefit trade-off. While we focus on $(k+1)^{t h}$ price auctions, the results can be transferred to more general mechanisms by the revenue equivalence theorem for multi-unit auctions in Engelbrecht-Wiggans (1988) in the case of continuous distributions.

\subsection{Information Release and the Dispersive Order}

This section illustrates how measures of dispersion allow to study the effects of information release in auctions. We provide an overview of existing results and point out their limitations by an example.

Intuitively, providing bidders with more information should increase the variability in their estimated valuations. The posterior distribution $F$ should thus be more variable (or "dispersed") than the prior $G$. In their analysis of information release, Ganuza and Penalva (2010) study two notions of dispersion, an ordering between $F$ and $G$ in the convex order, and an ordering of $F$ and $G$ in the dispersive order. These are defined as follows. ${ }^{7}$

\section{Definition 1}

(i) $F$ is more variable than $G$ in the convex order, $F \succeq_{c o n v} G,{ }^{8}$ if $E\left[X_{1}\right]=E\left[Y_{1}\right]$ and

$$
E\left[\left(X_{1}-k\right)^{+}\right] \geq E\left[\left(Y_{1}-k\right)^{+}\right] \text {for all } k \in \mathbb{R} .
$$

\footnotetext{
${ }^{7}$ For background on these two orders, see Chapters 3.A and 3.B of Shaked and Shanthikumar (2007). Our definitions follow their Theorem 3.A.1 and Formula 3.B.1.

${ }^{8}$ For our purposes, it proves to be more convenient to formulate stochastic orders on the level of distribution functions and not on the level of random variables as is done, e.g., in Shaked and Shanthikumar (2007).
} 
(ii) $F$ is more variable than $G$ in the dispersive order, $F \succeq_{\text {disp }} G$, if

$$
F^{-1}(p)-F^{-1}(q) \geq G^{-1}(p)-G^{-1}(q) \quad \text { for all } 0<q<p<1
$$

An ordering in the convex order is a weak requirement closely related to second-order stochastic dominance. It is satisfied in many models of information release. Under the assumption that $F \succeq_{\text {conv }} G$, Ganuza and Penalva show that releasing information increases expected welfare and, with sufficiently many bidders, the expected revenue in the auction. ${ }^{9}$ Both results follow from the intuition that increasing the variability of valuations tends to increase the highest valuations.

In order to control differences between overall welfare and seller's revenues, stronger orderings need to be imposed. Ganuza and Penalva rely on the dispersive order. $F$ dominates $G$ in the dispersive order if all pairs of quantiles lie further apart under $F$ than under $G$. As we will see below, this is a rather rigid requirement which is violated in many models of information release. The next lemma summarizes their results on information release in auctions under the assumption that $F \succeq_{\text {disp }} G{ }^{10}$

Lemma 2 Assume $F \succeq_{\text {disp }} G$ and $k=1$.

(i) Bidders' aggregate rents increase when information is released,

$$
E\left[X_{1: n}-X_{2: n}\right] \geq E\left[Y_{1: n}-Y_{2: n}\right]
$$

(ii) A welfare maximizing seller has a stronger incentive to release information than a revenue maximizing seller,

$$
E\left[X_{1: n}-Y_{1: n}\right] \geq E\left[X_{2: n}-Y_{2: n}\right]
$$

(iii) The expected welfare generated by the auction increases more strongly when the number of bidders increases under information release than when no information is released,

$$
E\left[X_{1: n}-X_{1: n-1}\right] \geq E\left[Y_{1: n}-Y_{1: n-1}\right] .
$$

\footnotetext{
${ }^{9}$ These results are their Theorems 3 and 5. A generalization to the $k$ object case, relying on their techniques and results by de la Cal and Cárcamo (2006) on majorization of order statistics is straightforward and omitted here.

${ }^{10}$ The four parts of Lemma 2 correspond to Proposition 6, Theorem 7, Theorem 4 and Theorem 6 of Ganuza and Penalva (2010).
} 
(iv) The seller's expected payoff increases more strongly when the number of bidders increases under information release than when no information is released,

$$
E\left[X_{2: n}-X_{2: n-1}\right] \geq E\left[Y_{2: n}-Y_{2: n-1}\right]
$$

All four results rely on comparisons of differences of order statistics, so-called spacings. Technically, they stem from the following fact about the dispersive order. ${ }^{11}$

Lemma 3 Let $F \succeq_{\text {disp }} G$. Then for all $k<n$

$$
E\left[X_{k: n}-X_{k+1: n}\right] \geq E\left[Y_{k: n}-Y_{k+1: n}\right]
$$

and

$$
E\left[X_{k: n}-X_{k: n-1}\right] \geq E\left[Y_{k: n}-Y_{k: n-1}\right] .
$$

In the remainder of this section, we illustrate a setting which does not fall under Lemma 2 and which leads to the opposite economic implications.

\section{Example 1}

Assume that bidders' true valuations are distributed uniformly on [0,1]. Bidders do not know their true valuations. They only know whether their valuation is below $2 / 3$ or not. By releasing information, the seller can furnish bidders with the additional information whether their valuations lie below or above $1 / 3$. Consequently, the a priori distribution $G$ puts a mass of $2 / 3$ on the value $1 / 3$ and the remaining mass on $5 / 6{ }^{12}$ The a posteriori distribution $F$ is a uniform distribution on $1 / 6,1 / 2$ and $5 / 6$. Notice first that $F$ and $G$ are not comparable in the dispersive order. When moving from $G$ to $F$ the lowest third of probability mass moves downwards from $1 / 3$ to $1 / 6$ while the middle third moves upwards from $1 / 3$ to $1 / 2$. The upper quantiles do not react to the information release. Therefore, the lower two-thirds of probability mass are indeed more dispersed under $F$ than under $G$. Yet the upper two-thirds lie more closely together. When working with information partitions, information release will always lead to such ambiguous effects and thus preclude a direct application of the dispersive order.

As Lemma 2 is not applicable in our example, we compare welfare and seller's

\footnotetext{
${ }^{11}$ The first claim of Lemma 3 follows from Theorem 3.B.41 of Shaked and Shanthikumar (2007). The second claim follows from the first and formula (6) below.

${ }^{12}$ For a more detailed introduction of this model, see Section 4.2 .
} 
revenues by a direct calculation,

$E\left[X_{1: n}-X_{2: n}\right]=\frac{1}{9} n\left(\frac{2}{3}\right)^{n-1}\left(1+\left(\frac{1}{2}\right)^{n-2}\right) \quad$ and $\quad E\left[Y_{1: n}-Y_{2: n}\right]=\frac{1}{6} n\left(\frac{2}{3}\right)^{n-1}$

For $n=2$, we obtain results similar to parts (i) and (ii) of Lemma 2. For $n=3$, welfare and seller's revenues react equally strongly. With four or more bidders, the results are reversed. Bidders' aggregate rents decrease when information is released. Thus a revenue maximizing seller has a stronger incentive to release information than a welfare maximizing one. ${ }^{13}$

In our example, information affects bidders with intermediate valuations more strongly than bidders with high valuations. This renders the auction more competitive. In particular, information release does not increase the differences between high order statistics. If we look at restrictions of $F$ and $G$ to sufficiently high quantiles, we see that, in a sense, information release reduces dispersion.

Definition 2 For $p \in(0,1)$ define the restriction of $F$ to its quantiles higher than $p$ as the cumulative distribution function

$$
F_{>p}(x)= \begin{cases}\frac{F(x)-p}{1-p} & x \geq F^{-1}(p) \\ 0 & x<F^{-1}(p)\end{cases}
$$

and define $G_{>p}(x)$ analogously. ${ }^{14}$

Consider the distributions $F_{>1 / 3}$ and $G_{>1 / 3} . \quad F_{>1 / 3}$ is the uniform distribution on $\{1 / 2,5 / 6\}$ while $G_{>1 / 3}$ is the uniform distribution on $\{1 / 3,5 / 6\}$. Unlike $F$ and $G$ themselves, these restrictions can be compared in the dispersive order. Yet it is the distribution without information release which is more dispersed, $G_{>\frac{1}{3}} \succeq_{\text {disp }} F_{>\frac{1}{3}}$. Since higher quantiles dominate the behavior of high order statistics with sufficiently many bidders, this observation explains the reversal of Lemma 2. Indeed, we will see in Proposition 6 and Theorem 1 that a dispersive ordering between $F$ and $G$ above some quantile is essentially a sufficient condition for whether Lemma 2 holds or whether it is reversed.

\footnotetext{
${ }^{13}$ As we will see in greater generality in Section 4.2, parts (iii) and (iv) of the lemma are also reversed with sufficiently many bidders.

${ }^{14}$ Notice that the definition is such that if $F$ has an atom on $F^{-1}(p)$, i.e., $F\left(F^{-1}(p)\right)=q>p$ then $F_{>p}(x)$ has an atom of size $(q-p) /(1-p)$ on $F^{-1}(p)$.
} 


\section{Dispersion Criteria for Order Statistics}

As seen in Lemma 3, the dispersive order implies a control over all spacings of order statistics while the outcomes of auctions depend only on the highest few. This motivates us to introduce the $k$-dispersion orders which are specifically designed to control spacings of the $k$ highest order statistics. Example 1 shows that a clear monotonicity behavior of these spacings may only develop with sufficiently many bidders. To capture these situations, we introduce the weaker $k$ - $m$-dispersion orders. These allow to control the behavior of the $k$ highest order statistics in auctions with more than $k+m$ bidders. The goal of both families of stochastic orders is to focus on the properties of a distribution which are most relevant for the auction's outcomes, and not to impose more restrictions than needed.

\subsection{The $k$-Dispersion Orders}

This section introduces the family of $k$-dispersion orders, compares them with other orders, and develops their implications.

Definition 3 ( $k$-Dispersion) For an integer $k \geq 1, F$ is more dispersed than $G$ in the $k$-dispersion order, $F \succeq_{k} G$, if

$$
\int_{p}^{1}(1-u)^{k} d F^{-1}(u) \geq \int_{p}^{1}(1-u)^{k} d G^{-1}(u)
$$

for all $p \in(0,1)$.

This definition is inspired by the following representation of spacings in terms of the quantile function, see, e.g., Kadane (1971),

$$
E\left[X_{k: n}-X_{k+1: n}\right]=\left(\begin{array}{l}
n \\
k
\end{array}\right) \int_{0}^{1} u^{n-k}(1-u)^{k} d F^{-1}(u) .
$$

The $k$-dispersion order $\succeq_{k}$ allows to control spacings of neighboring order statistics independently of $n$ as is shown in Propositions 2 and 3 below. In Section 5, we demonstrate its applicability to order statistics that lie further apart as well as to normalized spacings. 
$\succeq_{k}$ is a genuine stochastic order in that it is transitive: ${ }^{15}$ For three distribution functions $F, G$, and $H, F \succeq_{k} G$ and $G \succeq_{k} H$ imply $F \succeq_{k} H$. While the 1dispersion order coincides with the excess wealth order, ${ }^{16}$ the $k$-dispersion orders for $k>1$ appear to be novel. ${ }^{17}$ Like the excess wealth order, all $k$-dispersion orders are location independent, i.e., $F \succeq_{k} G$ remains fulfilled if either of the two distributions is shifted by a constant.

Proposition 1 sets $k$-dispersion into context. The dispersive order is stronger (and thus less broadly applicable) than all $k$-dispersion orders. For instance, it is a necessary condition for the dispersive order that $F^{-1}$ and $G^{-1}$ cross only once. $k$ dispersion does not rely on such a single-crossing condition.

Within the family of $k$-dispersion orders, $(k+1)$-dispersion implies $k$-dispersion. The convex order can generally not be compared to $k$-dispersion and the dispersive order as it is not location independent: $F \succeq_{\text {conv }} G$ can only hold if $F$ and $G$ have the same mean. Under the assumption that $F$ and $G$ share the same mean, the convex order is implied by each of the other orderings. Yet the convex order itself is not strong enough to control spacings of order statistics.

\section{Proposition 1}

(i) If $F \succeq_{\text {disp }} G$ then $F \succeq_{k} G$ for all $k \geq 1$.

(ii) If $F \succeq_{k+1} G$ then $F \succeq_{k} G$ for all $k \geq 1$.

(iii) If $E\left[X_{1}\right]=E\left[Y_{1}\right]$ and $F \succeq_{k} G$ then $F \succeq_{\text {conv }} G$ for all $k \geq 1$.

Proposition 2 demonstrates the suitability of $k$-dispersion for controlling spacings of high order statistics. The result combines Proposition 1 (ii) with Proposition 3.4 of Li and Shaked (2004).

Proposition 2 If $F \succeq_{k} G$ for some $k<n$ then for all $i \leq k$

$$
E\left[X_{i: n}-X_{i+1: n}\right] \geq E\left[Y_{i: n}-Y_{i+1: n}\right]
$$

Next, we extend this result to the other class of spacings of order statistics where we vary $n$ while keeping $i$ fixed. The key observation is that the two types of spacings differ only by a combinatorial factor which is not distribution-dependent.

\footnotetext{
${ }^{15}$ This separates $k$-dispersion from some single-crossing criteria for dispersion such as the rotation criterion of Johnson and Myatt (2006).

${ }^{16}$ See Shaked and Shanthikumar (2007) for background on the excess wealth order.

${ }^{17}$ The definition is motivated by an observation of Li and Shaked (2004), see Proposition 2 below.
} 
Proposition 3 If $F \succeq_{k} G$ for some $k<n$ then for all $i \leq k$

$$
E\left[X_{i: n}-X_{i: n-1}\right] \geq E\left[Y_{i: n}-Y_{i: n-1}\right]
$$

Since $k$-dispersion orders are location independent, we cannot expect to obtain results comparing $E\left[X_{k: n}\right]$ and $E\left[Y_{k: n}\right]$ without further assumptions. Under the additional requirement that $F$ and $G$ share the same mean, results of this type can be derived from the fact that $k$-dispersion implies the convex order.

\subsection{The $k$ - $m$-Dispersion Orders}

While the $k$-dispersion orders are weaker than the dispersive order, there are still many economically interesting examples for which they are too rigid. For instance, in Example 1 monotonicity of spacings sets in only with sufficiently many bidders. Building on $k$-dispersion, we therefore introduce the weaker class of $k$ - $m$-dispersion orders. These imply the results of Propositions 2 and 3 under the additional restriction that the number of bidders is sufficiently large, namely $n>k+m$.

Definition 4 ( $k$-m-Dispersion) For integers $k \geq 1$ and $m \geq 0, F$ is more dispersed than $G$ in the $k$-m-dispersion order, $F \succeq_{k, m} G$, if

$$
\int_{p}^{1} u^{m}(1-u)^{k} d F^{-1}(u) \geq \int_{p}^{1} u^{m}(1-u)^{k} d G^{-1}(u)
$$

for all $p \in(0,1)$.

The $k$ - $m$-dispersion orders are location-independent and transitive. $k$-0-dispersion coincides with our previous $k$-dispersion. Compared to $k$-dispersion, the increasing function $u^{m}$ in the integrand shifts attention into the right tail of the distribution. With many bidders, the behavior at this tail is crucial for an auction's outcomes. Proposition 4 summarizes the central properties of $k-m$-dispersion. The proposition generalizes Propositions 2 and 3.

\section{Proposition 4}

(i) If $F \succeq_{k, m} G$ for some $k$ and $m$ with $k+m<n$ then for all $i \leq k$

$$
E\left[X_{i: n}-X_{i+1: n}\right] \geq E\left[Y_{i: n}-Y_{i+1: n}\right] .
$$


(ii) If $F \succeq_{k, m} G$ for some $k$ and $m$ with $k+m<n$ then for all $i \leq k$

$$
E\left[X_{i: n}-X_{i: n-1}\right] \geq E\left[Y_{i: n}-Y_{i: n-1}\right]
$$

To put the $k$-m-dispersion orders into context we add the following result in the spirit of Proposition 1.

\section{Proposition 5}

(i) If $F \succeq_{k, m} G$ then $F \succeq_{k, m+1} G$ for all $k \geq 1$ and for all $m \geq 0$.

(ii) If $F \succeq_{k+1, m} G$ then $F \succeq_{k, m} G$ for all $k \geq 1$ and for all $m \geq 0$.

(iii) If $k, m \geq 1$ and $E\left[X_{1}\right]=E\left[Y_{1}\right]$ then $F \succeq_{k, m} G \nRightarrow F \succeq_{\text {conv }} G$ and $F \succeq_{\text {conv }} G \nRightarrow$ $F \succeq_{k, m} G$.

Accordingly, increasing $m$ renders the $k$ - $m$-dispersion order less rigid. All $k$ - $m$ dispersion orders are weaker than the $k$-dispersion order and, consequently, the dispersive order. Unlike $k$-dispersion, $k$ - $m$-dispersion is not comparable to the convex order if $F$ and $G$ have the same mean. Indeed, in the application to information partitions in Section 4.2, we find that $F \succeq_{\text {conv }} G$ is always satisfied together with either $F \succeq_{k, m} G$ or $G \succeq_{k, m} F$. The dispersive order is not applicable in these examples.

The following alternative sufficient condition for $k$ - $m$-dispersion is useful, e.g., when working with discrete distributions. The condition relies on the dispersive order between restrictions of $F$ and $G$ to high quantiles as introduced in Definition 2.

Proposition 6 Suppose there exists $p \in(0,1)$ such that $F_{>p} \succeq_{\text {disp }} G_{>p}$ and there exist $q_{1}, q_{2} \in(p, 1)$ with $F^{-1}\left(q_{2}\right)-F^{-1}\left(q_{1}\right)>G^{-1}\left(q_{2}\right)-G^{-1}\left(q_{1}\right)$. Then for any $k$ there exists $m$ such that $F \succeq_{k, m} G$.

In the proposition, the condition involving $q_{1}$ and $q_{2}$ ensures that the comparison in the dispersive order holds, in a sense, strictly. A direct consequence is the following corollary which states that for continuous distributions on a bounded support $F \succeq_{k, m}$ $G$ holds for sufficiently large $m$ if the density of $F$ is smaller than the density of $G$ near the tops of the respective supports.

Corollary 1 Suppose $F$ and $G$ are continuous with bounded supports $\left[a_{F}, b_{F}\right]$ and $\left[a_{G}, b_{G}\right]$ and possess continuous density functions $f$ and $g$. If there exists $\delta>0$ such 
that $0<f(x)<g(y)$ for all $x \in\left[b_{F}-\delta, b_{F}\right]$ and $y \in\left[b_{G}-\delta, b_{G}\right]$ then for any $k$ there exists $m$ such that $F \succeq_{k, m} G$.

\section{Information Release in Multi-Object Auctions}

\subsection{The General Case}

This section applies $k$ - and $k$-m-dispersion to information release in $k$ object auctions. Theorem 1 generalizes Lemma 2. It provides conditions for welfare reacting more strongly to information than seller's revenues, as well as conditions for the opposite situation. Furthermore, it covers the cases in which sufficiently many bidders need to take part in order to arrive at clear-cut results.

\section{Theorem 1}

(i) If $F \succeq_{k, m} G$ and $n>k+m$, then bidders' aggregate rents increase when information is released.

(ii) If $F \succeq_{k, m} G$ and $n>k+m$, then a welfare maximizing seller has a stronger incentive to release information than a revenue maximizing seller.

(iii) If $F \succeq_{k, m} G$ and $n>k+m$, then the welfare generated by the auction increases more strongly when the number of bidders increases under information release than when no information is released.

(iv) If $F \succeq_{k+1, m} G$ and $n>k+1+m$, then the expected selling price and the seller's payoff increase more strongly when the number of bidders increases under information release than when no information is released.

(v) The conclusions of (i-iii) are reversed if $G \succeq_{k, m} F$ and $n>k+m$. The conclusion of (iv) is reversed if $G \succeq_{k+1, m} F$ and $n>k+1+m$.

Thus, in the setting $k=1$ and $m=0$ of Ganuza and Penalva, the excess wealth order is sufficient for (i) to (iii) while the stronger 2-dispersion order is needed for (iv). We need to require stronger dispersion orders when the number of objects $k$ increases. An immediate consequence of (ii) is that if information release is costly then for intermediate cost levels a welfare maximizer releases information while a revenue maximizer does not. Finally, while the results may only hold with sufficiently many bidders, they are more than asymptotic results. For given $k, F$ and $G, k-m$-dispersion provides an explicit criterion for determining the value of $m$. 


\subsection{Information Partitions}

When information release takes the form of increasingly finer information partitions, Theorem 1 yields a complete characterization of information release with sufficiently many bidders. If information release increases the highest valuation estimate, the requirements of claims (i) to (iv) of the theorem are fulfilled. If the highest valuation estimate is unaffected by information release, the four claims are reversed.

Assume that bidders' true valuations are distributed according to a continuous distribution function $H$ with a strictly positive density $h$ on an interval $[a, b]$ with $a \geq 0$ and $a<b \leq \infty$. Denote by $\left(\beta_{i}\right)_{i}$ an ordered and strictly increasing subsequence of $(a, b)$ with $B>0$ elements. Thus, $\beta_{1}$ and $\beta_{B}$ are the lowest and highest values in the sequence. Without information release, bidders only know for each of the values $\beta_{i}$ whether their valuations lie above or below. Accordingly, the distribution $G$ of valuation estimates assigns probability

$$
H\left(\beta_{i}\right)-H\left(\beta_{i-1}\right) \quad \text { to the estimate } \frac{\int_{\beta_{i-1}}^{\beta_{i}} x h(x) d x}{H\left(\beta_{i}\right)-H\left(\beta_{i-1}\right)}
$$

with the obvious modifications for $\beta_{1}$ and $\beta_{B}$.

Information release is modeled such that the seller increases the number of values for which bidders know whether their valuation lies above or below. The sequence $\left(\beta_{i}\right)_{i}$ is thus replaced by another ordered and strictly increasing sequence $\left(\alpha_{i}\right)_{i}$ with $A>B$ elements. $\left(\beta_{i}\right)_{i}$ is a subsequence of $\left(\alpha_{i}\right)_{i}$. The distribution $F$ of posterior valuation estimates is derived from $\left(\alpha_{i}\right)_{i}$ analogously to (5).

Proposition 7 shows that for any $k, F$ and $G$ are always comparable in the $k-m$ dispersion order for sufficiently large $m$.

\section{Proposition 7}

(i) If $\alpha_{A}=\beta_{B}$, then for any $k$ there exists an $m$ such that $G \succeq_{k, m} F$.

(ii) If $\alpha_{A}>\beta_{B}$, then for any $k$ there exists an $m$ such that $F \succeq_{k, m} G$.

Whether $F$ or $G$ is more dispersed thus depends on whether information release affects the highest valuation estimates or not. If $\alpha_{A}=\beta_{B}$, the bidders with the highest valuation estimates are not affected by information release. The auction thus becomes more competitive such that the reverses of claims (i-iv) of Theorem 1 hold 
with sufficiently many bidders. If $\alpha_{A}>\beta_{B}$, information release further differentiates the valuation estimates of the highest valuation bidders. Consequently, the auction becomes less competitive and the four claims of Theorem 1 hold with sufficiently many bidders.

\subsection{Heterogeneous Signal Quality}

The quality of a signal may depend on the bidder's type. In this section, we provide a model that allows to study such a heterogeneity in signal quality in a multi-object auction context. We start with the following classical set-up. Bidders receive a noisy signal which is identical to their valuation with some probability and pure noise otherwise. With homogeneous signal quality, this is the truth-or-noise model introduced in Lewis and Sappington (1994) and applied, e.g., by Johnson and Myatt (2006), Ganuza and Penalva (2010), and Shi (2012). We study a variation of this model which captures heterogeneity in signal qualities. The probability of the signal being correct is different for bidders with high versus low valuations. Possible interpretations include information which is more vital to bidders with low valuations than to bidders with high ones (or vice versa), or, more generally, information which is more precise in some respects than in others.

Bidders' true valuations $Z_{i}$ are independent and uniformly distributed on $[0,1]$. Each agent receives an independent signal $S_{i}$ which is either equal to $Z_{i}$ or equal to $U_{i}$ where $U_{i}$ is independent of $Z_{i}$ and also uniformly distributed on $[0,1]$. There are numbers $\theta, p_{L}, p_{H} \in(0,1)$ such that the probability of $S_{i}=Z_{i}$ is $p_{L}$ for $Z_{i} \leq \theta$ and $p_{H}$ for $Z_{i}>\theta$. Signal quality thus depends on whether the true valuation is above or below $\theta$. We denote by $G$ the distribution of valuation estimates which follows from this specification of $\theta, p_{L}$ and $p_{H}$.

In this model, releasing more information corresponds to improvements in the quality of the signals. It can thus take three basic forms, an increase in $p_{H}$, an increase in $p_{L}$ or a shift of $\theta$ such that more agents have the higher signal quality. In the following, we refer to these three possibilities as an $H$-increase in information, an $L$-increase in information, and a $T$-increase in information. ${ }^{18}$ We denote by $F$ the distribution of valuation estimates which arises from either of these increases in the amount, or

\footnotetext{
${ }^{18}$ For the case of a $T$-increase, more information is released if $p_{H}>p_{L}$ and $\theta$ decreases, or if $p_{H}<p_{L}$ and $\theta$ increases. When $p_{H}=p_{L}$, changes in $\theta$ have no effect. We thus implicitly assume $p_{L} \neq p_{H}$ when speaking of a $T$-increase in information.
} 
quality, of information. In particular, we say that $F$ differs from $G$, e.g., through an $H$-increase in information if the two distributions are based on the same values of $p_{L}$ and $\theta$ but if $F$ has a higher value of $p_{H}$ than $G$.

In order to study the impact of information release, we need to establish what the distributions $G$ and $F$ look like. The probability $q_{L}$ of observing a signal below $\theta$ is given by

$$
q_{L}=P\left(S_{i} \leq \theta\right)=\theta p_{L}+\theta^{2}\left(1-p_{L}\right)+\theta(1-\theta)\left(1-p_{H}\right)=: p_{1}+p_{2}+p_{3},
$$

where the three summands $p_{j}$ correspond to the cases where $S_{i}=Z_{i} \leq \theta$, where $S_{i}, Z_{i} \leq \theta$ but $S_{i} \neq Z_{i}$, and where $S_{i} \leq \theta$ but $Z_{i}>\theta$. Analogously, we have

$q_{H}=P\left(S_{i}>\theta\right)=(1-\theta) p_{H}+(1-\theta)^{2}\left(1-p_{H}\right)+\theta(1-\theta)\left(1-p_{L}\right)=: p_{4}+p_{5}+p_{6}$.

The valuation estimate (and bid) of a bidder who received the signal realization $s \leq \theta$ is thus given by

$$
e_{L}(s)=\frac{1}{q_{L}}\left(s p_{1}+\frac{\theta}{2} p_{2}+\frac{1+\theta}{2} p_{3}\right)
$$

where the pre-factors of $p_{2}$ and $p_{3}$ are the means of uniform distributions on $[0, \theta]$ and $(\theta, 1]$. Similarly, an agent who received $s>\theta$ has the estimate

$$
e_{H}(s)=\frac{1}{q_{H}}\left(s p_{4}+\frac{1+\theta}{2} p_{5}+\frac{\theta}{2} p_{6}\right)
$$

Denote by $u(\cdot \mid I)$ the density of a uniform distribution on the interval $I$. Since signals remain uniformly distributed conditional on lying above or below $\theta$, the distribution of valuation estimates $G$ is a mixture of two uniform distributions and its density $g$ is given by $g(y)=q_{L} u\left(y \mid I_{L}\right)+q_{H} u\left(y \mid I_{H}\right)$ where

$$
I_{L}=\left[e_{L}(0), e_{L}(\theta)\right] \text { and } I_{H}=\left(e_{H}(\theta), e_{H}(1)\right]
$$

In this model, an increase in the amount of information does not necessarily imply a higher dispersion in the sense of the dispersive order. ${ }^{19}$ Moreover, higher values

\footnotetext{
${ }^{19}$ For instance, for $p_{L}=\theta=0.25$ and $p_{H}=0.1$, there is a gap between the two parts of the support $I_{L}$ and $I_{H}$. Improving signal quality by increasing $p_{H}$ to 0.25 closes this gap, $e_{L}(\theta)=$ $e_{H}(\theta)$, so that some quantiles lie more closely together than before, thus ruling out an ordering in the dispersive order.
} 
of the signal realization do not necessarily imply higher valuation estimates. Such a lack of monotonicity can occur if $\theta$ is sufficiently large so that $Z_{i}<\theta$ can still correspond to a rather high valuation, and if signal realizations below $\theta$ are more reliable than those above, $p_{L} \gg p_{H}$. For the auction, we need to determine whether the overall highest bids come from bidders with the highest possible signals (near 1 ), or from bidders with signals near $\theta$. This motivates the following definition of monotonicity at the top (MT).

Definition 5 The tuple $\left(p_{L}, p_{H}, \theta\right)$ satisfies monotonicity at the top (MT) if $e_{H}(1)>$ $e_{L}(\theta)$. The tuple $\left(p_{L}, p_{H}, \theta\right)$ violates $(\mathrm{MT})$ if $e_{H}(1)<e_{L}(\theta)$.

The next lemma provides an explicit equivalent condition and some illustrations of (MT). (MT) holds if high signals are more reliable than low ones, or if the overall reliability of signals is sufficiently high while the threshold $\theta$ is low. (MT) is violated if high signals are sufficiently unreliable, and if the threshold $\theta$ is sufficiently high.

\section{Lemma 4}

(i) (MT) is equivalent to

$$
0<S\left(p_{L}, p_{H}, \theta\right)=p_{L}+p_{H}+p_{L} p_{H}+\theta^{2} p_{L}^{2}-(1-\theta)^{2} p_{H}^{2}-2 \theta p_{L}-2 \theta p_{L} p_{H}
$$

(ii) (MT) is satisfied if $p_{H} \geq p_{L}$.

(iii) (MT) is satisfied if $(1-\theta)\left(p_{L}+p_{H}\right) \geq 1$.

(iv) For any $p_{L} \in(0,1)$, (MT) is violated if $p_{H}$ is sufficiently small and $\theta$ is sufficiently large.

The next two propositions characterize the effects of the three types of information release, first for the case where (MT) holds and then for the case where it is violated. We indicate whether $F \succeq_{k, m} G$ or vice versa for sufficiently high $m$. The results on auctions then follow directly from Theorem 1 .

Proposition 8 Suppose $\left(p_{L}, p_{H}, \theta\right)$ satisfy (MT).

(i) If $F$ differs from $G$ through a sufficiently small L-increase or T-increase in information, then for any $k$ there exists $m$ such that $F \succeq_{k, m} G$.

(ii) If $F$ differs from $G$ through a sufficiently small $H$-increase in information and if $p_{H}<\theta^{-1}-p_{L}$, then for any $k$ there exists $m$ such that $F \succeq_{k, m} G$. 
(iii) If $F$ differs from $G$ through a sufficiently small $H$-increase in information and if $p_{H}>\theta^{-1}-p_{L}$, then for any $k$ there exists $m$ such that $G \succeq_{k, m} F$.

In the proposition, "a sufficiently small increase" means that the increase leaves condition (MT) intact and, in cases (ii) and (iii), also the additional restriction on $p_{H}$. Increasing the amount of information through changes in $p_{L}$ or $\theta$ thus relaxes competition among sufficiently many bidders, i.e., assertions (i)-(iv) of Theorem 1 apply. In contrast, if $\theta, p_{L}$ and $p_{H}$ are sufficiently high, ${ }^{20}$ a further increase in $p_{H}$ induces a fiercer competition at the top and implies the reversals of assertions (i)(iv). In the latter case a further increase in $p_{H}$ leads to more bidders learning about their very high valuations. If the overall signal quality is already high, this effect dominates the welfare enhancing effects of information release such as a further differentiation of beliefs at the top. ${ }^{21}$ Finally, we investigate the situation where (MT) is violated so that the highest bids come from bidders with signals slightly below $\theta$.

Proposition 9 Suppose $\left(p_{L}, p_{H}, \theta\right)$ violate (MT). If $F$ differs from $G$ through a sufficiently small $H$-increase, $L$-increase or $T$-increase in information, then for any $k$ there exists $m$ such that $F \succeq_{k, m} G$.

Thus, if (MT) is violated and there are sufficiently many bidders, assertions (i)-(iv) of Theorem 1 hold for all three types of information release. Small amounts of information always soften competition at the top.

Our analysis describes which kind of information release appeals more to welfaremaximizing versus revenue-maximizing sellers. Another question is whether information release actually enhances welfare and the seller's revenue or not. In the information partitions model of Section 4.2, welfare and seller's revenue always increase in response to information release when there are sufficiently many bidders. In the model of this section, effects can be more intricate. With sufficiently many bidders, the question is equivalent to the question whether the upper end of the support $u=\max \left(e_{L}(\theta), e_{H}(1)\right)$ increases in response to information release. When

\footnotetext{
${ }^{20}$ Notice that $p_{H}>\theta^{-1}-p_{L}$ can only hold if the right hand side is smaller than 1 , i.e., if $\left(1+p_{L}\right) \theta>1$. To see that cases (ii) and (iii) of the proposition are both compatible with (MT), consider $p=p_{L}=p_{H}>\frac{1}{2}$. Then (MT) holds by Lemma 4 and whether we are in case (ii) or (iii) depends on whether $\theta<(2 p)^{-1} \in(0,1)$ or not.

${ }^{21}$ In particular, the effect which leads to a reversal of Theorem 1 in this model is distinct from the one we observed in the case of information partitions. There, the increased competition at the top was due to a further differentiation of intermediate valuation estimates.
} 
(MT) is satisfied, $H$ - and $L$-increases in information always lead to an increase in $u=e_{H}(1)$ and thus to higher welfare and seller's revenue with sufficiently many bidders. $^{22}$

\section{$5 \quad$ Further Applications}

This section sketches extensions of our analysis to other economic contexts, like matching markets, and the control of differences in low realizations which is important for risk management and reliability theory. Via $k$-dispersion, we can compare increments of expected order statistics $E\left[X_{k: n}\right]$ that are next to each other with regard to $k$ or $n$. In this section, we show that $k$-dispersion serves as a tool for controlling differences of order statistics that lie further apart as well, and describe where this control can be applied.

Proposition 10 If $F \succeq_{k} G$ for some $k<n$ then for all $i \leq k$ and all $l>i$

$$
E\left[X_{i: n}-X_{l: n}\right] \geq E\left[Y_{i: n}-Y_{l: n}\right]
$$

Proposition 10 characterizes which degree of $k$-dispersion is needed in order to compare specific differences of order statistics. For example, the 1-dispersion order allows to contrast differences between first and third order statistics across distributions. A similar comparison of the second and third order statistics requires the stronger 2-dispersion order. The proposition generalizes the main result of Kochar, Li and $\mathrm{Xu}(2007)^{23}$ which treats the case $k=1$.

A direct consequence of Proposition 10 is that it allows to compare sums of spacings of order statistics: $F \succeq_{k} G$ implies that

$$
\sum_{j=i}^{l-1} E\left[X_{j: n}-X_{j+1: n}\right] \geq \sum_{j=i}^{l-1} E\left[Y_{j: n}-Y_{j+1: n}\right]
$$

\footnotetext{
${ }^{22}$ For $T$-increases and for the case where (MT) is violated, the behavior of $u$ is more complex and a detailed discussion is beyond the scope of this paper. The results of Theorem 1 remain valid when $u$ decreases in response to information release but one might want to reinterpret (ii), e.g., in terms of incentives to prevent leakage of information.

${ }^{23}$ Kochar, Li and Xu apply their result to the study of one object $k^{\text {th }}$ price auctions. This part of their analysis is problematic from the viewpoint of game-theoretic auction theory since it relies on the assumption that bidders truthfully bid their valuations independently of the auction format.
} 
for all $i \leq k$ and all $n>l>i$. Proposition 11 provides similar results for normalized spacings of order statistics.

Proposition 11 If $F \succeq_{k} G$ for some $k<n$ then for all $i \leq k$ and all $l>i$

$$
\sum_{l=i}^{m} l E\left[X_{l: n}-X_{l+1: n}\right] \geq \sum_{l=i}^{m} l E\left[Y_{l: n}-Y_{l+1: n}\right]
$$

The case $k=1$ generalizes a result of Barlow and Proschan (1966) which is a key ingredient of Hoppe, Moldovanu and Sela (2009)'s analysis of matching markets. In the latter paper, women and men can invest into costly presents in order to improve their matching outcomes (and thus, e.g., match with a partner that is ranked better than the partner they would obtain otherwise). The inequality of Proposition 11 allows to study the comparative statics of signaling costs and welfare in this marriage market. Barlow and Proschan (1966) rely on the convex transform order which is stronger than the excess wealth order when $F$ and $G$ have the same mean. ${ }^{24}$ Proposition 11 shows that the results of Hoppe, Moldovanu, and Sela hold under weaker requirements on the distributions.

Regarding the spacings of the $k$ lowest order statistics, one can define the family of $\bar{k}$-dispersion orders given by

$$
F \succeq_{\bar{k}} G \quad \Leftrightarrow \quad \int_{0}^{p} u^{k} d F^{-1}(u) \geq \int_{0}^{p} u^{k} d G^{-1}(u) \quad \forall p \in(0,1)
$$

For example, expected differences in quality for the worst, second to worst, third to worst, etc. product out of a production series can be compared through these orders. All arguments for this family of orders are parallel to those we obtained for the $k$-dispersion orders. Like the 1-dispersion order, the $\overline{1}$-dispersion order coincides with a familiar stochastic order, namely, with the location independent risk order of Jewitt (1989).

\section{A Proofs}

\section{Proof of Proposition 1}

To see (i), notice that $F \succeq_{\text {disp }} G$ implies that the measure $\nu$ given by $d \nu(u)=$

\footnotetext{
${ }^{24}$ Shaked and Shanthikumar (2007), formula (4.B.3) shows that the convex transform order implies the star order. Li (2005), Remark 2.7, shows that the star order implies the excess wealth order if $F$ and $G$ share the same mean.
} 
$d\left(F^{-1}(u)-G^{-1}(u)\right)$ is non-negative, so that integrals of non-negative functions against $\nu$ are non-negative. Thus (2) holds for all $p$. (ii) is shown as follows: Lemma 7.1 of Chapter 4 of Barlow and Proschan (1981) states that for any signed measure $\nu$ on $\mathbb{R}^{+}$and any non-decreasing, non-negative function $h$

$$
\int_{p}^{\infty} d \nu(u) \geq 0 \quad \forall p>0 \Rightarrow \int_{0}^{\infty} h(u) d \nu(u) \geq 0 .
$$

Applying this result with $d \nu(u)=(1-u)^{k+1} d\left(F^{-1}(u)-G^{-1}(u)\right)$ shows that $F \succeq_{k+1} G$ implies

$$
\int_{0}^{1} h(u)(1-u)^{k+1} d F^{-1}(u) \geq \int_{0}^{1} h(u)(1-u)^{k+1} d G^{-1}(u)
$$

for any non-decreasing, non-negative $h$. Applying this inequality to all members of the family of non-decreasing functions $\left(h_{q}\right)_{q \in(0,1)}$ defined by $h_{q}(u)=(1-u)^{-1} 1_{\{u \geq q\}}$ yields

$$
\int_{q}^{1}(1-u)^{k} d F^{-1}(u) \geq \int_{q}^{1}(1-u)^{k} d G^{-1}(u) \quad \forall q \in(0,1)
$$

and thus $F \succeq_{k} G$. (iii) follows from the fact that $F \succeq_{k} G$ implies $F \succeq_{1} G$ by (ii), and from the fact that $\succeq_{1}$ is the excess wealth order so that we can apply Formula 3.C.8 of Shaked and Shanthikumar (2007).

\section{Proof of Proposition 2}

By Assertion (ii) of Proposition 1, it is sufficient to consider the case $k=i$. By (3), it is sufficient to show that

$$
\int_{0}^{1} u^{n-k}(1-u)^{k} d F^{-1}(u) \geq \int_{0}^{1} u^{n-k}(1-u)^{k} d G^{-1}(u) .
$$

This inequality follows from the definition (2) of the $k$-dispersion order by applying like in the proof of Proposition 1 - Lemma 7.1 of Chapter 4 of Barlow and Proschan (1981) to the signed measure $\nu$ given by $d \nu(u)=(1-u)^{k} d\left(F^{-1}(u)-G^{-1}(u)\right)$ and to the non-decreasing function $h(u)=u^{n-k}$.

\section{Proof of Proposition 3}

Again, by Assertion (ii) of Proposition 1, it is sufficient to consider the case $k=i$. Rewriting Relation 1 from David (1970, p. 45) into our notation yields

$$
E\left[X_{k: n}\right]-E\left[X_{k: n-1}\right]=\frac{k}{n}\left(E\left[X_{k: n}\right]-E\left[X_{k+1: n}\right]\right) .
$$


Thus we can apply Proposition 2 and conclude that $F \succeq_{k} G$ implies

$$
\begin{aligned}
E\left[X_{k: n}\right]-E\left[X_{k: n-1}\right] & =\frac{k}{n}\left(E\left[X_{k: n}\right]-E\left[X_{k+1: n}\right]\right) \\
& \geq \frac{k}{n}\left(E\left[Y_{k: n}\right]-E\left[Y_{k+1: n}\right]\right)=E\left[Y_{k: n}\right]-E\left[Y_{k: n-1}\right] .
\end{aligned}
$$

\section{Proof $^{25}$ of Proposition 4}

By Proposition 5 (ii) we can focus on the case $i=k$. The proof of (i) is entirely parallel to the one of Proposition 2 except that we choose $d \nu(u)=u^{m}(1-u)^{k} d\left(F^{-1}(u)-\right.$ $\left.G^{-1}(u)\right)$ and $h(u)=u^{n-k-m}$. (ii) follows from (i) and (6).

\section{Proof of Proposition 5}

The proof of (i) is entirely parallel to the one of Proposition 1 (ii) except that we choose $d \nu(u)=u^{m}(1-u)^{k+1} d\left(F^{-1}(u)-G^{-1}(u)\right)$. The same is true for the proof of (ii) where we choose $d \nu(u)=u^{m}(1-u)^{k} d\left(F^{-1}(u)-G^{-1}(u)\right)$ and $h_{q}(u)=u 1_{\{u \geq q\}}$. For (iii), notice that Proposition 7 provides a class of examples where $E\left[X_{1}\right]=E\left[Y_{1}\right]$, $F \succeq_{\text {conv }} G$ is satisfied together with either $F \succeq_{k, m} G$ or $G \succeq_{k, m} F$ for some $m$.

\section{Proof of Proposition 6}

Choose the measure $\nu$ as $d \nu(u)=(1-u)^{k} d\left(F^{-1}(u)-G^{-1}(u)\right)$. We have to show that there exists $m$ such that

$$
L(r)=\int_{r}^{1} u^{m} d \nu(u)
$$

is non-negative for all $r \in(0,1)$. By assumption, the measure $\nu$ is nonnegative over $[p, 1]$. This proves the claim for $r \geq p$. For $r<p$ consider the decomposition

$$
L(r)=\int_{r}^{p} u^{m} d \nu(u)+\int_{p}^{q_{1}} u^{m} d \nu(u)+\int_{q_{1}}^{q_{2}} u^{m} d \nu(u)+\int_{q_{2}}^{1} u^{m} d \nu(u) .
$$

The second and fourth integrals are non-negative by assumption. The first integral we can bound from below by replacing $\nu$ by its negative part $\nu^{-}$which exists by the Hahn decomposition:

$$
L(r) \geq \int_{r}^{p} u^{m} d \nu^{-}(u)+\int_{q_{1}}^{q_{2}} u^{m} d \nu(u)
$$

\footnotetext{
${ }^{25}$ The logical contingencies between Propositions $4-7$ are as follows: Proposition $6 \Rightarrow$ Proposition $7 \Rightarrow$ Proposition $5 \Rightarrow$ Proposition 4 .
} 
Since these are integrals with respect to a negative and a positive measure, we can further bound them from below as follows:

$$
L(r) \geq p^{m}\left(\int_{0}^{p} d \nu^{-}(u)\right)+q_{1}^{m}\left(\int_{q_{1}}^{q_{2}} d \nu(u)\right) .
$$

The first term in brackets is finite since the supports of $F$ and $G$ are bounded from below. The second term in brackets is strictly positive by our assumption on $q_{1}$ and $q_{2}$. Since $q_{1}>p$, it thus follows that $L(r) \geq 0$ for sufficiently large $m$. Since the final lower bound is independent of $r$, this choice of $m$ is the same for all $r$.

\section{Proof of Corollary 1}

The fact that $f$ is strictly smaller than $g$ at the top of the support implies that there exists a threshold $p$ such that all pairs of quantiles greater than the $p$-quantile lie strictly further apart under $F$ than under $G$. This implies $F_{>p} \succeq_{\text {disp }} G_{>p}$ and the claim follows from Proposition 6.

\section{Proof of Theorem 1}

Observe that we can write bidders' aggregate rents after information release as

$$
\sum_{j=1}^{k} E\left[X_{j: n}-X_{k+1: n}\right]=\sum_{j=1}^{k} j E\left[X_{j: n}-X_{j+1: n}\right]
$$

To the expression on the right hand side we can apply Proposition 4 and conclude

$$
\sum_{j=1}^{k} E\left[X_{j: n}-X_{k+1: n}\right] \geq \sum_{j=1}^{k} E\left[Y_{j: n}-Y_{k+1: n}\right]
$$

which is (i). Rearranging this inequality yields

$$
\sum_{j=1}^{k} E\left[X_{j: n}-Y_{j: n}\right] \geq E\left[k X_{k+1: n}-k Y_{k+1: n}\right]
$$

which proves (ii). The welfare gains from adding an additional bidder when releasing information are given by $\sum_{j=1}^{k} E\left[X_{j: n}-X_{j: n-1}\right]$. This is greater than the corresponding quantity with $Y$ in place of $X$ by Proposition 4. This shows (iii). The claim about the expected selling price in (iv) follows from observing that Proposition 4 yields

$$
E\left[X_{k+1: n}-X_{k+1: n-1}\right] \geq E\left[Y_{k+1: n}-Y_{k+1: n-1}\right]
$$


provided that $F \succeq_{k+1} G$. The statement about the seller's payoff follows by multiplying this inequality with $k$. (v) follows by exchanging the roles of $F$ and $G$.

\section{Proof of Proposition 7}

Denote by $\alpha^{*}$ the largest element of $\left(\alpha_{i}\right)_{i}$ which is not included in $\left(\beta_{i}\right)_{i}$ and set $p=H\left(\alpha^{*}\right)$. We prove (i) by showing that $G_{>p} \succeq_{\text {disp }} F_{>p}$ and then invoking Proposition 6 . Denote by $\beta_{+}^{*}>\beta_{-}^{*}$ the upper and lower neighbors of $\alpha^{*}$ in the sequence $\left(\beta_{i}\right)_{i}$. Observe that the distributions $F_{>p}$ and $G_{>p}$ are both discrete distributions concentrated on a finite number of values. In particular, since the two partitions are identical from $\beta_{+}^{*} \in\left(\alpha_{i}\right)_{i}$ on, the two distributions are identical except for the lowest value. For $F_{>p}$, the lowest possible realization $l_{F}$ is the conditional mean of $H$ over the set $\left[\alpha^{*}, \beta_{+}^{*}\right]$, while for $G_{>p}$ this lowest realization is the conditional mean $l_{G}$ over $\left[\beta_{-}^{*}, \beta_{+}^{*}\right]$. Both occur with the same positive probability $\left(H\left(\beta_{+}^{*}\right)-H\left(\alpha^{*}\right)\right) /(1-p)$. Clearly, we have $l_{F}>l_{G}$. Since this difference between the lowest realizations is the only difference of $F_{>p}$ and $G_{>p}$, it follows directly that $G_{>p} \succeq_{\text {disp }} F_{>p}$. Since all probabilities are strictly positive, we can also guarantee existence of $q_{1}$ and $q_{2}$ as required by Proposition 6 .

The proof of (ii) proceeds similarly by showing that $F_{>p} \succeq_{\text {disp }} G_{>p}$. We set $p=$ $H\left(\beta_{B}\right)$. Then $G_{>p}$ is a degenerate distribution which takes as its only value the conditional mean of $H$ over $\left[\beta_{B}, b\right] . \quad F_{>p}$ takes at least two values with positive probability, since the sequence $\left(\alpha_{i}\right)$ contains at least one element which is greater than $\beta_{B}$. We thus have $F_{>p} \succeq_{\text {disp }} G_{>p}$.

\section{Proof of Lemma 4}

A direct calculation reveals that

$$
e_{H}(1)-e_{L}(\theta)=\frac{S\left(p_{L}, p_{H}, \theta\right)}{2\left(1+\left(p_{L}-p_{H}\right)(1-\theta)\right)\left(1+\left(p_{H}-p_{L}\right) \theta\right)} .
$$

Since $\left|p_{H}-p_{L}\right|<1$, the denominator is always positive and (i) follows. For (ii), note that $S$ is concave in $p_{H}$ so it suffices to verify $S\left(p_{L}, p_{L}, \theta\right)=2 p_{L}(1-\theta)>0$ and

$$
S\left(p_{L}, 1, \theta\right)=2 p_{L}+2 \theta-4 p_{L} \theta-\theta^{2}\left(1-p_{L}^{2}\right)>0 .
$$

The last claim follows from the facts that $S\left(p_{L}, 1, \theta\right)$ is concave in $\theta$ and that $S\left(p_{L}, 1,1\right)=\left(1-p_{L}\right)^{2}>0$ as well as $S\left(p_{L}, 1,0\right)=2 p_{L}>0$. For (iii), notice 
that $S$ can be written as

$$
S\left(p_{L}, p_{H}, \theta\right)=p_{L}(1-\theta)\left(1+p_{H}\right)+p_{H}+\theta^{2} p_{L}^{2}-(1-\theta)^{2} p_{H}^{2}-\theta p_{L}-\theta p_{L} p_{H}
$$

Applying in the first summand the assumed inequality $p_{L}(1-\theta) \geq 1-p_{H}(1-\theta)$, and rearranging, shows that $S$ is bounded from below by the function

$$
M\left(p_{L}, p_{H}, \theta\right)=-p_{H}^{2}(2-\theta)(1-\theta)+p_{H}\left(1+\theta-p_{L} \theta\right)+1-p_{L} \theta\left(1-p_{L} \theta\right) .
$$

Since $M$ is concave in $p_{H}, M>0$ follows from the positivity of $M\left(p_{L}, 0, \theta\right)=$ $1-p_{L} \theta\left(1-p_{L} \theta\right)$ and $M\left(p_{L}, 1, \theta\right)=\theta\left(4-2 p_{L}-\theta+p_{L}^{2} \theta\right)$. For (iv), it suffices to notice that $S$ is continuous and $S\left(p_{L}, 0,1\right)=-p_{L}\left(1-p_{L}\right)<0$.

\section{Proof of Proposition 8}

Since $G$ is a mixture of uniform distributions, it suffices to study how the value of the density at the highest valuation estimates reacts to changes in the parameters and then to apply Corollary 1 . Since (MT) holds, the value of the density at the top is given by

$$
T\left(p_{L}, p_{H}, \theta\right)=\frac{q_{H}}{e_{H}(1)-e_{H}(\theta)}=\frac{\left(1+\theta\left(p_{H}-p_{L}\right)\right)^{2}}{p_{H}} .
$$

The relevant derivatives of $T$ are given by

$$
\frac{\partial T}{\partial p_{L}}=-\frac{2 \theta\left(1+\left(p_{H}-p_{L}\right) \theta\right)}{p_{H}}, \frac{\partial T}{\partial \theta}=\frac{2\left(p_{H}-p_{L}\right)\left(1+\left(p_{H}-p_{L}\right) \theta\right)}{p_{H}}
$$

and

$$
\frac{\partial T}{\partial p_{H}}=-\frac{\left(1-\left(p_{H}+p_{L}\right) \theta\right)\left(1+\left(p_{H}-p_{L}\right) \theta\right)}{p_{H}^{2}} .
$$

Since $\left|p_{H}-p_{L}\right| \theta<1, \frac{\partial T}{\partial p_{L}}$ is always negative, implying that $F \succeq_{k, m} G$ for sufficiently large $m$ by Corollary 1. $\frac{\partial T}{\partial \theta}$ is negative when $p_{L}>p_{H}$ and positive when $p_{H}>p_{L}$, implying that $F \succeq_{k, m} G$ follows if $\theta$ is shifted into the direction of the smaller probability. The sign of $\frac{\partial T}{\partial p_{H}}$ depends on the sign of $1-\left(p_{H}+p_{L}\right) \theta$ as indicated in the proposition.

\section{Proof of Proposition 9}

We only point out the differences to the proof of Proposition 8. Since (MT) is 
violated, the density at the top is now given by

$$
T\left(p_{L}, p_{H}, \theta\right)=\frac{q_{L}}{e_{L}(\theta)-e_{L}(0)}=\frac{\left(1+(1-\theta)\left(p_{L}-p_{H}\right)\right)^{2}}{p_{L}} .
$$

The derivatives with respect to $\theta, p_{H}$ and $p_{L}$ are given by

$$
\frac{\partial T}{\partial p_{H}}=-\frac{2(1-\theta)\left(1+\left(p_{L}-p_{H}\right)(1-\theta)\right)}{p_{L}}, \quad \frac{\partial T}{\partial \theta}=\frac{2\left(p_{H}-p_{L}\right)\left(1+\left(p_{L}-p_{H}\right)(1-\theta)\right)}{p_{L}}
$$

and

$$
\frac{\partial T}{\partial p_{L}}=-\frac{\left(1-\left(p_{H}+p_{L}\right)(1-\theta)\right)\left(1+\left(p_{L}-p_{H}\right)(1-\theta)\right)}{p_{L}^{2}} .
$$

The signs of the derivatives follow like in Proposition 8 except that we do not distinguish cases because a violation of $(\mathrm{MT})$ implies $\left(p_{H}+p_{L}\right)(1-\theta)<1$ by Lemma 4.

\section{Proof of Propositions 10 and 11}

It is convenient to give a combined proof of the two propositions. By Assertion (ii) of Proposition 1, it is sufficient to consider the case $k=i$. From (3) we obtain that

$$
E\left[X_{k: n}-X_{l: n}\right]=\int_{0}^{1} \sum_{j=k}^{l-1}\left(\begin{array}{l}
n \\
j
\end{array}\right) u^{n-j}(1-u)^{j} d F^{-1}(u)
$$

and

$$
\sum_{j=k}^{l} j E\left[X_{j: n}-X_{j+1: n}\right]=\int_{0}^{1} \sum_{j=k}^{l-1} j\left(\begin{array}{l}
n \\
j
\end{array}\right) u^{n-j}(1-u)^{j} d F^{-1}(u) .
$$

Obviously, the right hand sides coincide up to the factor $j$ in the second sum. In the following, we denote this factor by $\varphi(j)$ and consider the choices $\varphi(j)=1$ and $\varphi(j)=j$. Now we claim the following:

Claim: For both, $\varphi(j)=1$ and $\varphi(j)=j$, there exists a non-decreasing function $h$ such that we can write

$$
\sum_{j=k}^{l-1} \varphi(j)\left(\begin{array}{l}
n \\
j
\end{array}\right) u^{n-j}(1-u)^{j}=h(u)(1-u)^{k}
$$

Provided that this claim is true, the desired inequality follows from the definition (2) of the $k$-dispersion order by applying - like in the proof of Proposition 1 - Lemma 7.1 of Chapter 4 of Barlow and Proschan (1981) to the signed measure $\nu$ given by 
$d \nu(u)=(1-u)^{k} d\left(F^{-1}(u)-G^{-1}(u)\right)$ and to the non-decreasing function $h$ identified in the claim: We obtain

$$
\int_{0}^{1} h(u) d \nu(u) \geq 0
$$

and thus

$$
\int_{0}^{1} \sum_{j=k}^{l-1} \varphi(j)\left(\begin{array}{l}
n \\
j
\end{array}\right) u^{n-j}(1-u)^{j} d F^{-1}(u) \geq \int_{0}^{1} \sum_{j=k}^{l-1} \varphi(j)\left(\begin{array}{l}
n \\
j
\end{array}\right) u^{n-j}(1-u)^{j} d G^{-1}(u) .
$$

Thus it remains to prove the claim. Since we can write

$$
\sum_{j=k}^{l-1} \varphi(j)\left(\begin{array}{c}
n \\
j
\end{array}\right) u^{n-j}(1-u)^{j}=(1-u)^{k} \sum_{j=k}^{l-1} \varphi(j)\left(\begin{array}{c}
n \\
j
\end{array}\right) u^{n-j}(1-u)^{j-k}
$$

this amounts to proving that

$$
h(u)=\sum_{j=k}^{l-1} \varphi(j)\left(\begin{array}{l}
n \\
j
\end{array}\right) u^{n-j}(1-u)^{j-k}
$$

is increasing in $u$ for our two choices of $\varphi(j)$. The key idea is to rewrite $h$ in terms of a $\operatorname{Binomial}(n-k, 1-u)$ distribution. We can write

$$
h(u)=\sum_{j=0}^{l-k-1} \varphi(k+j)\left(\begin{array}{c}
n \\
k+j
\end{array}\right) u^{n-k-j}(1-u)^{j}=\sum_{j=0}^{n-k} \Psi(j)\left(\begin{array}{c}
n-k \\
j
\end{array}\right) u^{n-k-j}(1-u)^{j}
$$

where

$$
\Psi(j)=\varphi(k+j) \frac{\left(\begin{array}{c}
n \\
k+j
\end{array}\right)}{\left(\begin{array}{c}
n-k \\
j
\end{array}\right)} 1_{\{j<l-k\}}=\varphi(k+j) \frac{n \cdot \ldots \cdot(n-k+1)}{(j+k) \cdot \ldots \cdot(j+1)} 1_{\{j<l-k\}} .
$$

For our two choices of $\varphi$ which yield, respectively $\varphi(k+j)=1$ and $\varphi(k+j)=j+k$, $\Psi(j)$ is clearly a non-negative, non-increasing function. Now denote by $Z_{n-k, 1-u}$ a random variable distributed according to the $\operatorname{Binomial}(n-k, 1-u)$ distribution. From writing $h$ as

$$
h(u)=E\left[\Psi\left(Z_{n-k, 1-u}\right)\right]
$$

we can see that $h$ is non-decreasing in $u$ since $\Psi$ is non-increasing. 


\section{References}

[1] Barlow, Richard and Frank Proschan. Inequalities for Linear Combinations of Order Statistics from Restricted Families. Annals of Mathematical Statistics 37, 1593-1601, 1966.

[2] Barlow, Richard and Frank Proschan. Statistical Theory of Reliability and Life Testing. Holt, Rinehart and Winston, New York, 1981.

[3] Bergemann, Dirk and Martin Pesendorfer. Information structures in optimal auctions, Journal of Economic Theory 137, 580-609, 2007.

[4] Bergemann, Dirk and Juuso Välimäki. Information in Mechanism Design. In: Richard Blundell, Whitney Newey and Torsten Persson, eds., Proceedings of the 9th World Congress of the Econometric Society. Cambridge University Press, Cambridge, 186-221, 2006.

[5] Cal, Jesús de la and Javier Cárcamo. Stochastic orders and majorization of mean order statistics. Journal of Applied Probability 43, 704-712, 2006.

[6] David, Herbert A. Order Statistics. Wiley, New York, 1970.

[7] Engelbrecht-Wiggans, Richard. Revenue Equivalence in Multi-Object Auctions. Economics Letters 26, 15-19, 1988.

[8] Ganuza, Juan-José and José Penalva. Signal Orderings based on Dispersion and the Supply of Private Information in Auctions. Econometrica 78, 10071030, 2010.

[9] Hoppe, Heidrun C., Benny Moldovanu and Aner Sela. The Theory of Assortative Matching Based on Costly Signals. Review of Economic Studies 76, 253-281, 2009 .

[10] Jewitt, Ian. Choosing between risky prospects: the characterization of comparative statics results, and location independent risk. Management Science 35, 60-70, 1989

[11] Jia, Justin, Ronald M. Harstad and Michael H. Rothkopf. Information Variability Impacts in Auctions, Decision Analysis 7, 137-143, 2010. 
[12] Johnson, Justin and David Myatt. On the Simple Economics of Advertising, Marketing, and Product Design. American Economic Review 96, 756-784, 2006.

[13] Kadane, Joseph B. A Moment Problem for Order Statistics. Annals of Mathematical Statistics 42, 745-751, 1971.

[14] Kirkegaard, René. Ranking Asymmetric Auctions: Filling the Gap between a Distributional Shift and Stretch. Games and Economic Behavior 85, 60-69, 2014 .

[15] Kochar, Subhash, Xiaohu Li and Maochao Xu. Excess wealth order and sample spacings. Statistical Methodology 4, 385-392, 2007.

[16] Krishna, Vijay. Auction Theory. Academic Press, San Diego, 2002.

[17] Lewis, Tracy R., and David E. M. Sappington. Supplying information to facilitate price discrimination. International Economic Review 35, 309-327, 1994.

[18] Li, Xiaohu. A note on expected rent in auction theory. Operations Research Letters 33, 531-534, 2005.

[19] Li, Xiaohu and Moshe Shaked. The observed total time on test and the observed excess wealth. Statistics \& Probability Letters 68, 247-258, 2004.

[20] Mares, Vlad and Jeroen M. Swinkels. On the analysis of asymmetric first price auctions. Journal of Economic Theory 152, 1-40, 2014.

[21] Paul, Anand and Genaro Gutierrez. Mean sample spacings, sample size and variability in an auction-theoretic framework. Operations Research Letters 32, 103-108, 2004.

[22] Rösler, Anne-Katrin. Information Disclosure in Markets: Auctions, Contests and Matching Markets. Working Paper, University of Bonn, 2014.

[23] Shaked, Moshe and J. George Shanthikumar. Stochastic Orders. Springer, New York, 2007.

[24] Shi, Xianwen. Optimal auctions with information acquisition. Games and Economic Behavior 74, 666-686, 2012.

[25] Xu, Maochao and Xiaohu Li. Some Further Results on the Winner's Rent in the Second-Price Business Auction. Sankhya 70 A, 124-133, 2008. 


\section{Working Paper Series in Economics}

recent issues

No. 67 Nikolaus Schweizer and Nora Szech: Revenues and welfare in auctions with information release, April 2015

No. 66 Andranik Tangian: Decision making in politics and economics: 6. Empirically constructing the German political spectrum, April 2015

No. 65 Daniel Hoang and Martin Ruckes: The effects of disclosure policy on risk management incentives and market entry, November 2014

No. 64 Sebastian Gatzer, Daniel Hoang, Martin Ruckes: Internal capital markets and diversified firms: Theory and practice, November 2014

No. 63 Andrea Hammer: Innovation of knowledge intensive service firms in urban areas, October 2014

No. 62 Markus Höchstötter and Mher Safarian: Stochastic technical analysis for decision making on the financial market, October 2014

No. 61 Kay Mitusch, Gernot Liedtke, Laurent Guihery, David Bälz: The structure of freight flows in Europe and its implications for EU railway freight policy, September 2014

No. 60 Christian Feige, Karl-Martin Ehrhart, Jan Krämer: Voting on contributions to a threshold public goods game - an experimental investigation, August 2014

No. 59 Tim Deeken and Ingrid Ott: Integration as a spatial institution: Implications for agglomeration and growth, July 2014

No. 58 Mher Safarian: Erhaltungsgesetze für das Modell $M_{r}\left|G_{r}\right| 1 \mid \infty$ in der Klasse der konservativen Abfertigungsdisziplinen, July 2014

No. 57 Marten Hillebrand: Existence of bubbly equilibria in overlapping generations models with stochastic production, June 2014 